\title{
A saúde na região ribeirinha de Aquidauana, MS: conhecendo o perfil dos usuários acamados, de seus cuidadores e 0 acesso aos serviços de fisioterapia
}

\author{
Health in the riverside region of Aquidauana, MS: knowing the profile of \\ beddrien users, their caregivers and the access to physical therapy services
Salud en la región ribereña de Aquidauana, MS: conociendo el perfil de los usuarios, sus cuidadores y el acceso a los servicios de fisioterapia

\author{
Arthur de Almeida Medeiros ${ }^{1}$ \\ Micheli Silva Alves ${ }^{1}$ \\ Wilson de Souza Franco ${ }^{1}$
}

Recebido em 19/03/2020; revisado e aprovado em 30/07/2020; aceito em 16/09/2020

DOI: http://dx.doi.org/10.20435/inter.v22i2.2972

\begin{abstract}
Resumo: O conhecimento da realidade da população é primordial para a discussão sobre o cuidado em saúde. Assim, o objetivo deste estudo foi conhecer o perfil dos usuários acamados e de seus cuidadores na região ribeirinha de Aquidauana, Mato Grosso do Sul (MS), e avaliar o acesso aos serviços de fisioterapia. Trata-se de um estudo transversal, no qual foram realizadas entrevistas domiciliares com os usuários acamados e seus cuidadores moradores da região ribeirinha de Aquidauana, MS, assistidos pela Estratégia Saúde da Família. Participaram do estudo 14 usuários acamados, sendo a maioria mulheres (64,3\%), de nível socioeconômico classe D (78,6\%), que foram encaminhadas a algum serviço de reabilitação (78,6\%) e à fisioterapia, o serviço mais referenciado (81,8\%). As condições clínicas mais frequentes foram a senilidade $(35,7 \%)$ e a disfunção mental $(28,6 \%)$. Todos os usuários acamados possuíam cuidadores, sendo a maioria mulheres (64,3\%), com algum grau de parentesco, sendo mais frequentes as filhas $(28,6 \%)$ e as esposas $(21,4 \%)$, que não receberam orientação de como prestar o cuidado (57,1\%). Assim, os dados apontam que a maioria dos usuários acamados eram idosas, de baixo nível socioeconômico, com independência funcional total e que foram encaminhadas a serviços de reabilitação; e, entre os cuidadores, a maioria eram idosas, familiares e com algum nível de sobrecarga.
\end{abstract}

Palavras-chave: atenção primária à saúde; fisioterapia; vulnerabilidade em saúde; cuidadores; pessoa com limitação física.

Abstract: The knowledge of the population's reality is an essential stage for the discussion about health care. Thus this study aimed to get to know the profile of bedridden users and their caregivers in the riverside region of Aquidauana, Mato Grosso do Sul (MS), and to assess the access to physical therapy services. This is a cross-sectional study in which home interviews were conducted with bedridden users and their caregivers living in the Aquidauana, MS, riverside region, assisted by the Family Health Strategy. Fourteen bedridden users participated in the study, most of them women (64.3\%), of socioeconomic class D (78.6\%), who were referred to some rehabilitation service $(78.6 \%)$ and to physical therapy, which was the most referenced service (81.8\%). The most frequent clinical conditions were senility (35.7\%) and mental dysfunction (28.6\%). All bedridden users had caregivers, most of whom were women (64.3\%), with some degree of kinship, being daughters (28.6\%) and wives (21.4\%), which did not receive guidance on how to provide care (57.1\%). Thus the data indicate that the majority of bedridden users were elderly women, of low socioeconomic status, with total functional independence, and who were referred to rehabilitation services; and, among caregivers, the majority were elderly women, family members, and with some level of burden.

Keywords: primary health care; physical therapy specialty; health vulnerability; caregivers, disabled persons. Resumen: El conocimiento de la realidad de la población es esencial para la discusión sobre la atención de la salud. Por lo tanto, el objetivo de este estudio fue conocer el perfil de los usuarios encamados y sus cuidadores en la región ribereña de Aquidauana, Mato Grosso do Sul (MS), y evaluar el acceso a los servicios de fisioterapia. Este es un estudio transversal en el que se realizaron entrevistas domiciliarias con usuarios encamados y sus cuidadores que viven en la región ribereña de Aquidauana, MS, con la asistencia de la Estrategia de Salud Familiar. Participaron en el estudio 14 usuarios encamados, la mayoría de los cuales eran mujeres (64,3\%), de clase socioeconómica $D(78,6 \%)$, que fueron derivadas a algún servicio de rehabilitación $(78,6 \%)$ y a fisioterapia, el servicio más referenciado $(81,8 \%)$. Las condiciones clínicas más frecuentes fueron

\footnotetext{
${ }^{1}$ Universidade Federal de Mato Grosso do Sul (UFMS), Campo Grande, Mato Grosso do Sul, Brasil.
} 
senilidad (35.7\%) y disfunción mental (28.6\%). Todos los usuarios postrados en cama tenían cuidadores, la mayoría de los cuales eran mujeres (64,3\%), con cierto grado de parentesco, siendo hijas $(28,6 \%)$ y esposas $(21,4 \%)$ las más frecuentes, que no recibieron orientación sobre cómo ofrecer cuidados (57.1\%). Así, los datos indican que la mayoría de los usuarios encamados eran ancianas, de bajo nivel socioeconómico, con total independencia funcional y que fueron remitidas a servicios de rehabilitación; y, entre los cuidadores, la mayoría eran ancianas, familiares y con algún nivel de sobrecarga.

Palabras clave: atención primaria de salud; fisioterapia; vulnerabilidad en salud; cuidadores; personas con discapacidad.

\section{INTRODUÇÃO}

Estudar e compreender a condição de saúde das pessoas se constitui como etapa primordial para a formulação e o planejamento de políticas públicas de saúde. Sabe-se que, em algumas situações, as informações de fontes de dados secundárias - apesar de serem extremamente importantes - não são suficientes para subsidiar tomada de decisões (MALTA et al., 2008).

Neste sentido, a realização de pesquisas de base populacional se mostra de extrema relevância, pois permite conhecer o perfil de saúde de determinada população, além de possibilitar a avaliação de políticas públicas vigentes, em especial em grupos sociais e geográficos excluídos ou pouco estudados (GAMA et al., 2018). No Brasil, são escassos os estudos que buscaram conhecer a realidade da população ribeirinha, em especial da população ribeirinha do Pantanal.

O Pantanal é a maior planície inundável do planeta, com uma área aproximada de 150.335 $\mathrm{km}^{2}$, que se estende entre os estados de Mato Grosso (MT) e Mato Grosso do Sul (MS), sendo que a sua maior área (64,64\%) está em MS (INSTITUTO BRASILEIRO DE GEOGRAFIA E ESTATÍSTICA [IBGE], s.d.). O município de Aquidauana é um dos que mais contribuem para a formação da área do Pantanal, conjuntamente com Corumbá, Cáceres e Poconé (SILVA; ABDON, 1998).

Com o crescimento rápido e desorganizado da cidade de Aquidauana, associado ao aumento da população, a falta de suporte e de um projeto para o desenvolvimento urbano fez com que ocorresse, de forma gradativa e desordenada, ocupação das áreas ribeirinhas do município (JÓIA; ANUNCIAÇÃO, 2013).

A rotina da população ribeirinha está sob influência da dinâmica das águas, e a maior parte vive em condições sanitárias precárias. Para compreender o processo de adoecimento, é preciso conhecer a rotina de vida destes indivíduos para que se possa proporcionar o cuidado de maneira adequada às suas demandas e necessidades. Devido à dificuldade de acesso, principalmente na época de cheia e transbordamento dos rios, a procura aos serviços de saúde pelos ribeirinhos e a atuação das equipes de saúde na oferta do cuidado são prejudicadas.

Nesse contexto, é de extrema importância reconhecer esta população para elaboração de estratégias que fortaleçam o vínculo entre equipes de saúde e comunidade na busca da oferta do cuidado integral e resolutivo. Deste modo, este trabalho teve como objetivo descrever o perfil dos usuários acamados e de seus respectivos cuidadores, que moram na região ribeirinha de Aquidauana, MS, dentro do perímetro urbano do município, e avaliar o acesso ao serviço de fisioterapia por esses usuários.

A seguir, é apresentado o percurso metodológico desenvolvido para contemplar os objetivos propostos, e os principais achados desta pesquisa, que buscou caracterizar a situação de saúde da população que vive na região ribeirinha do Pantanal sul-mato-grossense. 


\section{METODOLOGIA}

Trata-se de um estudo transversal realizado na região ribeirinha da cidade de Aquidauana, MS, assistida pelas equipes da Estratégia Saúde da Família (ESF). O município possui cobertura de $100 \%$ da ESF e conta com 16 equipes de Saúde da Família (eSF).

O estudo foi realizado no período de julho a dezembro de 2017, e foram incluídos os usuários acamados e seus cuidadores, maiores de 18 anos, pertencentes à região ribeirinha com cobertura da ESF.

Considerou-se como acamada a pessoa restrita ao seu domicílio por qualquer dificuldade de locomover-se, por si só, a qualquer centro de atenção à saúde.

Por não existir um sistema de informação com o quantitativo de usuários acamados que considere o usuário acamado conforme a definição adotada para este estudo, optou-se por apresentar tal definição aos agentes comunitários de saúde (ACS), os quais puderam preencher um formulário com nome e endereço dos usuários que atendessem aos critérios estabelecidos.

Após o levantamento realizado pelos ACS, procedeu-se visita ao domicílio dos usuários indicados, momento em que foram apresentados o estudo e o Termo de Consentimento Livre e Esclarecido (TCLE). As entrevistas foram realizadas com os usuários que decidiram voluntariamente participar do estudo ou com os cuidadores dos usuários que não possuíam condição cognitiva para tal.

A coleta de dados se deu por um instrumento desenvolvido exclusivamente para esta pesquisa, o qual continha questões referentes às variáveis do usuário acamado: idade; sexo; condição clínica; nível socioeconômico - avaliado pelo Critério de Classificação Econômica do Brasil (CCEB); serviços ou profissionais de reabilitação aos quais foi encaminhado; Medida da Independência Funcional (MIF); se houve acesso ao serviço de fisioterapia; motivo pelo qual não teve acesso; tipo de serviço utilizado; se utilizava o serviço de fisioterapia no momento da entrevista; e motivo pelo qual não se encontrava em tratamento.

Em relação ao cuidador, foram elaboradas questões relacionadas a: idade; sexo; relação/ grau de parentesco com o usuário acamado; se havia recebido orientação de como prestar cuidado; e avaliação do nível de sobrecarga avaliada pela Burden Interview.

Os dados foram submetidos à análise descritiva, utilizando-se o programa SPSS, versão 23.0.

O estudo atendeu aos preceitos éticos para pesquisa em seres humanos, conforme prevê a Resolução do Conselho Nacional de Saúde n. 466/2012, recebendo parecer favorável do Comitê de Ética em Pesquisa com Seres Humanos da Universidade Federal de Mato Grosso do Sul (parecer n. 2.148.934).

\section{RESULTADOS}

A região ribeirinha de Aquidauana é dividida em duas microáreas, contudo somente uma das áreas encontrava-se assistida por um ACS, o qual identificou 12 usuários elegíveis para o estudo. Durante a realização das entrevistas, os próprios usuários identificaram mais três possíveis participantes da pesquisa. Ao final, a amostra foi constituída por 14 usuários acamados, visto que um usuário se recusou a participar.

Entre os usuários entrevistados, verificou-se que a média da idade foi de $63,38 \pm 22,83$ anos, sendo que a maioria foi do sexo feminino ( $n=9 ; 64,37 \%)$, nível socioeconômico classe D $(n=9 ; 64,3 \%)$ e encaminhada a algum serviço de reabilitação $(n=11 ; 78,6 \%)$. A condição 
clínica apresentada pelos entrevistados foi disfunção neurológica ( $n=2 ; 14,3)$, disfunção musculoesquelética ( $n=3 ; 21,4 \%)$, disfunção mental $(n=4 ; 28,6 \%)$ e senilidade $(n=5 ; 35,7 \%)$. Entre os usuários que foram encaminhados a algum serviço de reabilitação, constata-se que o serviço de fisioterapia foi o que mais recebeu demanda de encaminhamentos ( $n=9 ; 81,8 \%$ ). A média da medida da independência funcional, de acordo com cada segmento, foi: MIF Motora 63,00 \pm 31,21 pontos, MIF Cognitiva 24,00 $\pm 11,10$ pontos e MIF Total 87,00 \pm 39,31 pontos (Tabela 1).

Tabela 1 - Perfil dos usuários acamados assistidos pela Estratégia Saúde da Família na região ribeirinha de Aquidauana, MS. Aquidauana, 2017

\begin{tabular}{lcc}
\hline \multicolumn{1}{c}{ Variáveis } & $\mathbf{n .}$ & $\mathbf{\%}$ \\
\hline Sexo & & \\
$\quad$ Feminino & 9 & 64,3 \\
Masculino & 5 & 35,7 \\
Condição Clínica & & \\
$\quad$ Disfunção Neurológica & 2 & 14,3 \\
Disfunção Musculoesquelética & 3 & 21,4 \\
Disfunção Mental & 4 & 28,6 \\
Senilidade & 5 & 35,7 \\
Nível Socioeconômico & & \\
C & 4 & 28,6 \\
D & 9 & 64,3 \\
E & 1 & 7,1 \\
Foi encaminhado a algum serviço de reabilitação? & & \\
Sim & 11 & 78,6 \\
Não & 3 & 21,4 \\
Serviço de Reabilitação ao qual foi encaminhado & & \\
Fisioterapia & 9 & 81,8 \\
Fonoaudiologia & 2 & 18,1 \\
Psicologia & 1 & 9,1 \\
Nutrição & 2 & 18,1 \\
Terapia Ocupacional & 3 & 27,3 \\
Classificação da Funcionalidade & & \\
Dependência Total & 1 & 7,1 \\
Dependência Modificada - assistência de até 50\% & 2 & 14,3 \\
Dependência Modificada - assistência de até 25\% & 2 & 14,3 \\
Independência Total & 9 & 64,3 \\
\hline Fonte: Osautors & & \\
\hline
\end{tabular}

Fonte: Os autores.

Todos os usuários acamados apresentavam cuidador e, no grupo de cuidadores, verificouse que a média da idade foi de 60,13 \pm 10,19 anos, com predomínio de cuidadores do sexo feminino ( $n=9 ; 64,3 \%)$, sendo as filhas as mais frequentes $(n=4 ; 28,6 \%)$, seguidas das esposas $(n=3 ; 21,4 \%)$ e dos filhos $(n=3 ; 21,4 \%)$. A maioria dos cuidadores não recebeu orientação de como prestar o cuidado $(n=8 ; 57,1 \%)$ e apresentou média da escala do nível de sobrecarga de $31,88 \pm 21,08$ pontos (Tabela 2). 
Tabela 2 - Perfil dos cuidadores de usuários acamados assistidos pela Estratégia Saúde da Família na região ribeirinha de Aquidauana, MS. Aquidauana, 2017

\begin{tabular}{|c|c|c|}
\hline Variáveis & n. & $\%$ \\
\hline \multicolumn{3}{|l|}{ Sexo } \\
\hline Feminino & 9 & 64,3 \\
\hline Masculino & 5 & 35,7 \\
\hline \multicolumn{3}{|l|}{ Relação com o usuário } \\
\hline Filha & 4 & 28,6 \\
\hline Filho & 3 & 21,4 \\
\hline Mãe & 2 & 14,3 \\
\hline Esposa & 3 & 21,4 \\
\hline Neto & 1 & 7,1 \\
\hline Pai & 1 & 7,1 \\
\hline \multicolumn{3}{|c|}{ Recebeu orientações de como prestar o cuidado? } \\
\hline Não & 8 & 57,1 \\
\hline Sim & 6 & 42,9 \\
\hline \multicolumn{3}{|l|}{ Classificação do Nível de Sobrecarga } \\
\hline Ausência de Sobrecarga & 5 & 35,7 \\
\hline Sobrecarga Moderada & 6 & 42,9 \\
\hline Sobrecarga Moderada a Severa & 1 & 7,1 \\
\hline Sobrecarga Severa & 2 & 14,3 \\
\hline
\end{tabular}

Fonte: Os autores.

Foi possível observar que a maioria dos usuários encaminhados ao serviço de fisioterapia teve acesso a ele $(n=8 ; 88,9 \%)$. Contudo um usuário relatou não ter tido acesso por falta de vagas na rede de serviços. A respeito do tipo de serviço utilizado, percebe-se que o Sistema Único de Saúde (SUS) foi o responsável pela maioria dos atendimentos ( $n=6 ; 75,0 \%$ ). Quando questionados se ainda realizavam tratamento, todos os usuários relataram que não, e os motivos apresentados foram dificuldade de transporte/locomoção ( $n=4 ; 28,6 \%$ ), falta de vagas $(n=3$; $21,4 \%)$ e alta ( $n=1 ; 7,1 \%)$ (Tabela 3 ).

Tabela 3 - Conhecimento sobre o acesso aos serviços de fisioterapia pelos usuários acamados assistidos pela Estratégia Saúde da Família na região ribeirinha de Aquidauana, MS. Aquidauana, 2017

\begin{tabular}{llc}
\hline \multicolumn{1}{c}{ Variáveis } & n. & \% \\
\hline $\begin{array}{l}\text { Teve acesso ao serviço de fisioterapia? } \\
\text { Não }\end{array}$ & 1 & 11,1 \\
Sim & 8 & 88,9 \\
Motivo pelo qual não teve acesso $\quad$ & 1 & 100,0 \\
$\quad$ Falta de vagas & & \\
Tipo de serviço utilizado & 6 & 75,0 \\
$\quad$ SUS & 2 & 25,0 \\
$\quad$ Serviço Privado & 1 & 12,5 \\
$\quad$ Plano de Saúde & & 100,0
\end{tabular}




\begin{tabular}{lcc}
\hline \multicolumn{1}{c}{ Variáveis } & n. & \% \\
\hline Motivo do encerramento do atendimento & & \\
Dificuldade de locomoção/transporte & 4 & 28,6 \\
Falta de vagas & 3 & 21,4 \\
Alta & 1 & 7,1 \\
\hline \hline
\end{tabular}

Fonte: Os autores.

\section{DISCUSSÃO}

Considerando os escassos estudos como referência para identificar os usuários acamados ribeirinhos, mostra-se a necessidade de pesquisas desta natureza, a fim de que se possa construir uma base de dados para o planejamento e desenvolvimento de ações para o cuidado dessa população.

A população ribeirinha, mesmo vivendo em situações adversas, revela perfil compatível com a situação da saúde demonstrada nas pesquisas realizadas no Brasil, em que a transição demográfica acontece muito acelerada, caracterizando-se pelo envelhecimento da população e elevação na proporção de mulheres em relação aos homens (AMARAL et al., 2012; WORLD HEALTH ORGANIZATION [WHO], 2011; VIANA et al., 2013).

O nível socioeconômico evidencia que a maioria dos entrevistados pertence às classes econômicas mais inferiores, indicando que, embora tenha ocorrido um acréscimo na renda, pela implementação de programas sociais, as condições econômicas ainda são limitadas. Há uma grande variabilidade na fonte de renda da população ribeirinha, e esta variação se encontra diretamente relacionada aos períodos de safra e entressafra, visto que, em muitas situações, a família vive exclusivamente da atividade pesqueira e, no período do defeso biológico, recebe apenas o auxílio defeso, reduzindo, portanto, a sua fonte de arrecadação (LIMA; DORIA; FREITAS, 2012).

Uma situação de destaque observada na pesquisa refere-se ao considerável número de usuários com disfunção mental. O relatório mundial sobre os cuidados inovadores para condições crônicas aponta que a inclusão das disfunções mentais possibilita a ampliação dos conceitos tradicionais de condição crônica, visto que alguns distúrbios, como a depressão e a esquizofrenia, tornam-se crônicos pela necessidade de acompanhamento, monitoramento e gerenciamento por um longo período, e ainda as disfunções mentais podem gerar impactos pessoais, sociais e econômicos, levando, muitas vezes, a situações de incapacidade (ORGANIZAÇÃO MUNDIAL DA SAÚDE [OMS], 2003).

Quanto ao encaminhamento aos serviços de reabilitação, sabe-se que limitações importantes e/ou redução da força muscular e incapacidades funcionais são os principais motivos pela procura por serviços de saúde (ACIOLE; BATISTA, 2013), e resultados semelhantes foram observados em um estudo que identificou que alterações nas funções relacionadas ao tônus muscular, ao controle dos movimentos voluntários, às funções emocionais e às funções sexuais estiveram diretamente relacionadas aos encaminhamentos aos serviços de reabilitação em indivíduos após acometimento por acidente vascular encefálico (LUCENA et al., 2017).

Assim, tais situações podem explicar o elevado número de encaminhamentos ao serviço de fisioterapia identificado na presente pesquisa, uma vez que a fisioterapia se relaciona diretamente com a funcionalidade humana. Observa-se, ainda, que os serviços ofertados pelo SUS foram os que receberam a maioria das demandas, demonstrando a importância e a necessidade da estruturação da rede de atenção à saúde da pessoa com deficiência. 
Os entrevistados relataram que não estavam realizando tratamento em razão, principalmente, da dificuldade para deslocamento até o centro de reabilitação, demonstrando claramente a existência de uma barreira de acesso aos serviços, e que estes usuários se encontram sem acompanhamento especializado e com probabilidades de acentuação de suas incapacidades, devido à dificuldade de transporte e/ou locomoção. Um estudo realizado na capital do estado evidenciou que os usuários que realizavam acompanhamento fisioterapêutico deslocavam-se de ônibus, a pé ou de bicicleta, revelando que o acesso ao serviço se dava, sobretudo, pelos usuários que possuíam melhor funcionalidade (SILVA; SANTOS; BONILHA, 2014).

Estudos indicam que as pessoas com deficiência física ou mobilidade reduzida se deslocam, principalmente, utilizando carros particulares ou alugados (AMARAL et al., 2012; SILVA; SANTOS; BONILHA, 2014). Contudo é imprescindível destacar que a amostra deste estudo é constituída pela população ribeirinha com baixo poder aquisitivo e que, portanto, não dispõe de recursos financeiros para efetivação do deslocamento até o centro de reabilitação, ocasionando a descontinuidade no tratamento. Desta maneira, a existência de transporte adequado e adaptado, no município, constitui-se como fator de proteção para o acesso aos serviços de saúde (AMARAL; MOTTA; SILVA; ALVES, 2012).

Em um estudo que buscou avaliar a sobrecarga, a qualidade de vida e a presença de estresse em cuidadores assistidos pela ESF em Criciúma, Santa Catarina, os autores verificaram que a média da MIF total dos usuários assistidos por estes cuidadores foi de $70,2 \pm 40,1$ pontos (SOUZA et al., 2015). Já em outro estudo, que teve como objetivo principal identificar a dependência funcional de idosos e avaliar a sobrecarga de seus cuidadores, verificou-se que os idosos que

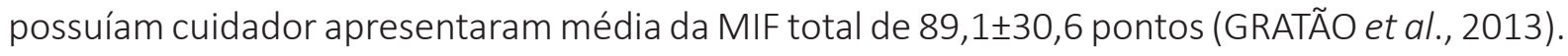
Em ambos os estudos citados, a média dos pontos obtidos na MIF apontou que os indivíduos avaliados apresentavam dependência modificada com necessidade de assistência de até 25\% nas atividades, resultados semelhantes ao desta pesquisa.

Contudo, ao realizar a estratificação da funcionalidade, observa-se que a maioria dos participantes foram classificados com independência total, assim, acredita-se que a condição de acamado entre os entrevistados na presente pesquisa esteja relacionada, principalmente, às más condições das vias públicas e da localização das residências, demonstrando que a situação de restrição e barreira ao acesso aos serviços de saúde, nestes casos, relaciona-se com as condições de infraestrutura de vias públicas, e não com a funcionalidade do usuário propriamente dito, visto que inadequações de acessibilidade dos meios de transporte público e as irregularidades nas calçadas e vias utilizadas para o deslocamento seguro e independente constituem-se como barreiras ao acesso aos serviços de saúde (SANTOS et al., 2017).

Quanto aos cuidadores, observou-se que a maioria são mulheres que possuem algum grau de parentesco com o acamado, e tal situação já tem sido reportada na literatura (COSTA et al., 2015; GRATÃO et al., 2013; HEDLER et al., 2016; LINO et al., 2016; MUNIZ et al., 2016). Nestes casos, são considerados cuidadores informais, pois quem assume a responsabilidade assistencial, na totalidade dos domicílios visitados, são os familiares, nos quais não recebem nenhuma remuneração e exercem a função por necessidade, carinho ou obrigação (LOUREIRO et al., 2013).

Em relação ao grau de parentesco, é tradicional e subconscientemente imposto que um familiar seja o cuidador, e na maioria dos casos os filhos veem esta situação como uma obrigação, já que, quando criança, foram cuidados por seus familiares (RICO-BLÁZQUEZ et al., 2014). Para 
além disto, há ainda a cultura de que a mulher é a responsável pelo cuidado, tanto dos filhos como da casa, e que, portanto, deve assumir tal responsabilidade.

Entretanto, na contemporaneidade, cada vez mais as mulheres têm assumido posições de destaque no mercado de trabalho, sem abandonar seu papel de cuidadora, e esta nova configuração tem contribuído para o surgimento de quadros de sobrecarga biopsicossocial (CARVALHO et al., 2015; PEREIRA et al., 2013).

Há relato na literatura de que, em muitas situações, a pessoa que presta o cuidado não se sente preparada para assumir tal função (BAPTISTA et al., 2012) e, associado a isso, observase neste estudo que a maioria dos entrevistados não recebeu orientações de como exercer a função de cuidador, uma situação já relatada em outras pesquisas (ANJOS et al., 2015; MUNIZ et al., 2016).

A falta de informações de como lidar com essa situação leva a consequências futuras, pois afeta as atividades pessoais do cuidador, levando à exaustão, estresse e perda de vínculos, como amizade, emprego, cuidado e higiene pessoal, lazer, relações afetivas etc. O despreparo para o papel de cuidador pode relacionar-se com situações de sobrecarga e surgimento de problemas de saúde (STACKFLETH et al., 2012).

Desempenhar atividades relacionadas ao bem-estar físico e psicossocial do paciente acamado ou restrito ao lar faz com que o cuidador passe a ter restrições em relação à sua vida social e, em muitos casos, até interferindo em sua vida conjugal, o que contribui para o surgimento da sobrecarga (LOUREIRO et al., 2013). As situações apresentadas podem, portanto, justificar os dados obtidos neste estudo, em que se observou que a maioria dos cuidadores apresentou algum grau de sobrecarga, sendo a maior parte identificada com sobrecarga moderada.

A sobrecarga imposta ao cuidador informal provoca uma perturbação física ou psicológica, resultante do lidar com a dependência motora ou mental do paciente (familiar) que necessita de sua atenção e dos seus cuidados. Para os cuidadores da região ribeirinha, esse processo de cuidar de um familiar acamado ou restrito ao lar é ininterrupto e quase sempre não pode ser revertido, em razão da dificuldade financeira e, na maioria das vezes, do fato de ser o único cuidador. Isso gera situações que podem levar à sobrecarga, como o reconhecimento da diminuição da qualidade de vida do paciente; limitação do tempo para realização de suas atividades; relação afetiva conflituosa entre cuidador e o sujeito que recebe os cuidados; e a falta de opção ou escolha para dividir ou compartilhar essa responsabilidade (PEREIRA et al., 2013).

As transformações ocorridas na vida do cuidador de pessoas acamadas podem contribuir significativamente para o comprometimento de sua saúde, principalmente pela limitação do exercício do autocuidado (PEREIRA et al., 2017).

Outro fator que pode aumentar a sobrecarga do cuidador na região ribeirinha é a dificuldade de acesso, que é agravada por fatores como falta de transporte público no município; distância entre o centro de reabilitação e o domicílio; e a inundação das ruas na época da cheia.

\section{CONCLUSÃO}

A presente pesquisa permitiu identificar que os usuários acamados residentes na região ribeirinha de Aquidauana são, em sua maioria, idosos, de baixo nível socioeconômico, e a avaliação da funcionalidade indicou que são pessoas com independência total, segundo a MIF. Quanto à condição clínica, observa-se o elevado número de usuários com disfunção mental. 
Todos os usuários acamados participantes da pesquisa possuíam cuidador, e estes eram pessoas idosas, familiares do usuário acamado - frequentemente, filhas ou esposas - que não receberam orientações de como prestar o cuidado. E a avaliação do nível de sobrecarga apontou que estes cuidadores apresentavam nível de sobrecarga moderada.

Verifica-se que a maioria dos usuários acamados foi encaminhada a algum serviço de reabilitação, sendo a fisioterapia o serviço mais referenciado. É importante destacar que os usuários, apesar de terem tido acesso aos serviços públicos de fisioterapia, haviam interrompido o tratamento diante da dificuldade de locomoção ou transporte, sugerindo, portanto, que a condição das vias públicas da região ribeirinha apresentou-se como barreira para o acesso aos serviços de saúde.

Ainda que os resultados desta pesquisa retratem uma realidade local, o presente estudo traz uma contribuição de extrema relevância para elaboração e implementação de ações e políticas públicas para o cuidado à população que vive na região ribeirinha do Pantanal sul-mato-grossense.

\section{REFERÊNCIAS}

ACIOLE, G. G.; BATISTA, L. H. Promoção da saúde e prevenção de incapacidades funcionais dos idosos na estratégia de saúde da família: a contribuição da fisioterapia. Saúde em debate, Rio de Janeiro, v. 37, n. 96, p. 10-9, jan./mar. 2013.

AMARAL, F. L. J. S.; HOLANDA, C. M. A.; QUIRINO, M. A. B.; NASCIMENTO, J. P. S.; NEVES, R. F.; RIBEIRO, K. S. Q. S.; ALVES, S. B. Acessibilidade de pessoas com deficiência ou restrição permanente de mobilidade ao SUS. Ciência e Saúde Coletiva, Rio de Janeiro, v. 17, n. 7, p. 1833-40, jul. 2012.

AMARAL, F. L. J. S.; MOTTA, M. H. A.; SILVA, L. P. G.; ALVES, S. B. Fatores associados com a dificuldade no acesso de idosos com deficiência aos serviços de saúde. Ciência e Saúde Coletiva, Rio de Janeiro, v. 17, n. 11, p. 2991-3001, nov. 2012.

ANJOS, K. F.; BOERY, R. N. S. O.; PEREIRA, R.; PEDREIRA, L. C.; VILELA, A. B. A.; SANTOS, V. C.; ROSA, D. O. S. Association between social support and quality of life of relative caregivers of elderly dependents. Ciência e Saúde Coletiva, Rio de Janeiro, v. 20, n. 5, p. 1321-30, maio 2015.

BAPTISTA, B. O.; BEUTER, M.; GIRARDON-PERLINI, N. M. O.; BRONDANI, C. M.; BUDÓ, M. L. D.; SANTOS, N. O. A sobrecarga do familiar cuidador no âmbito domiciliar: uma revisão integrativa da literatura. Revista Gaúcha de Enfermagem, Porto Alegre, v. 33, n. 1, p. 147-56, mar. 2012.

CARVALHO, D. P.; TOSO, B. R. G. O.; VIERA, C. S.; GARANHANI, M. L.; RODRIGUES, R. M.; RIBEIRO, L. F. C. Ser cuidador e as implicações do cuidado na atenção domiciliar. Texto e Contexto Enfermagem, Florianópolis, v. 24, n. 2, p. 450-8, abr./jun. 2015.

COSTA, T. F.; COSTA, K. N. F. M.; MARTINS, K. P.; FERNANDES, M. G. M.; BRITO, S. S. Burden over family caregivers of elderly people with stroke. Escola Anna Nery, Rio de Janeiro, v. 19, n. 2, p. 350-55, abr./jun. 2015.

GAMA, A. S. M.; FERNANDES, T. G.; PARENTE, R. C. P.; SECOLI, S. R. Inquérito de saúde em comunidades ribeirinhas do Amazonas, Brasil. Cadernos de Saúde Pública, Rio de Janeiro, v. 34, n. 2, p. 1-16, fev. 2018.

GRATÃO, A. C. M.; TALMELLI, L. F. S.; FIGUEIREDO, L. C.; ROSSET, I.; FREITAS, C. P.; RODRIGUES, R. A. P. Dependência funcional de idosos e a sobrecarga do cuidador. Revista da Escola de Enfermagem da USP, São Paulo, v. 47, n. 1, p. 137-44, fev. 2013. 
HEDLER, H. C.; FALEIROS, V. P.; SANTOS, M. J. S.; ALMEIDA, M. A. A. Representação social do cuidado e do cuidador familiar do idoso. Revista Katálysis, Florianópolis, v. 19, n. 1, p. 143-53, jan./jun. 2016.

INSTITUTO BRASILEIRO DE GEOGRAFIA E ESTATÍ́STICA [IBGE]. Território. IBGE, Rio de Janeiro, [s.l.], [s.d.]. Disponível em: https://brasilemsintese.ibge.gov.br/territorio.html. Acesso em 20 dez. 2019.

JÓIA, P. R.; ANUNCIAÇÃO, V. S. Inundações urbanas e vulnerabilidade socioespacial na cidade de Aquidauana. Geografia, Londrina, v. 22, n. 2, p. 5-23, maio/ago. 2013.

LIMA, M. A. L.; DORIA, C. R. C.; FREITAS, C. E. C. Pescarias artesanais em comunidades ribeirinhas na amazônia brasileira: perfil socioeconômico, conflitos e cenário da atividade. Ambiente e Sociedade, São Paulo, v. 15, n. 2, p. 73-90, maio/ago. 2012.

LINO, V. T. S.; RODRIGUES, N. C. P.; CAMACHO, L. A. B.; O'DWYER G.; LIMA, I. S.; ANDRADE, M. K. N.; ATIE, $S$. Prevalência de sobrecarga e respectivos fatores associados em cuidadores de idosos dependentes, em uma região pobre do Rio de Janeiro, Brasil. Cadernos de Saúde Pública, Rio de Janeiro, v. 32, n. 6, p. 1-14, jun. 2016.

LOUREIRO, L. S. N.; FERNANDES, M. G. M.; MARQUES, S.; NÓBREGA, M. M. L.; RODRIGUES, R. A. P. Sobrecarga de cuidadores familiares de idosos: prevalência e associação com características do idoso e do cuidador. Revista da Escola de Enfermagem da USP, São Paulo, v. 47, n. 5, p. 1133-40, out. 2013.

LUCENA, E. M. F.; RIBEIRO, K. S. Q. S.; MORAES, R. M.; NEVES, R. F.; BRITO, G. E. G.; SANTOS, R. N. L. C. Relationship between body functions and referral to rehabilitation post-stroke. Fisioterapia em Movimento, Curitiba, v. 30, n. 1, p. 141-50, jan./mar. 2017.

MALTA, D. C.; LEAL, M. C.; COSTA, M. F. L.; MORAIS NETO, O. L. Inquéritos Nacionais de Saúde: experiência acumulada e proposta para o inquérito de saúde brasileiro. Revista Brasileira de Epidemiologia, São Paulo, v. 11, n. supl. 1, p. 159-67, maio 2008.

MUNIZ, E. A.; FREITAS, C. A. S. L.; OLIVEIRA, E. N.; LACERDA, M. R. Grau de sobrecarga dos cuidadores de idosos atendidos em domicílio pela Estratégia Saúde da Família. Saúde em Debate, Rio de Janeiro, v. 40, n. 110, p. 172-82, jul./set. 2016.

ORGANIZAÇÃO MUNDIAL DA SAÚDE [OMS]. Cuidados inovadores para condições crônicas: componentes estruturais de ação. Brasília: OMS, 2003.

PEREIRA, L. T. S.; NOVAES, G. J.; MORAES, L.; BORGES, C. J.; SOUZA, M. R.; SILVA, L. A.; BARROS, P. S. Um olhar sobre a saúde das mulheres cuidadoras de idosos: desafios e possibilidades. Revista Kairós: Gerontologia, São Paulo, v. 20, n. 1, p. 277-297, 2017.

PEREIRA, R. A.; SANTOS, E. B.; FHON, J. R. S.; MARQUES, S.; RODRIGUES, R. A. P. Sobrecarga dos cuidadores de idosos com acidente vascular cerebral. Revista da Escola de Enfermagem da USP, São Paulo, v. 47, n. 1, p. 185-92, fev. 2013.

RICO-BLÁZQUEZ et al. CuidaCare: effectiveness of a nursing intervention on the quality of life's caregiver: cluster-randomized clinical trial. BMC Nursing, [s.I.], v. 13, n. 1, p. 1-10, 2014.

SANTOS, M. D.; SILVA, M. F.; VELLOZA, L. A.; POMPEU, J. E. Lack of accessibility in public transport and inadequacy of sidewalks: effects on the social participation of elderly persons with functional limitations. Revista Brasileira de Geriatria e Gerontologia, Rio de Janeiro, v. 20, n. 2, p. 161-74, mar./abr. 2017.

SILVA, M. A.; SANTOS, M. L. M.; BONILHA, L. A. S. Fisioterapia ambulatorial na rede pública de saúde de Campo Grande (MS, Brasil) na percepção dos usuários: resolutividade e barreiras. Interface, Botucatu, v. 18, n. 48, p. 75-86, 2014. 
SILVA, J. S. V.; ABDON, M. M. Delimitação do pantanal brasileiro e suas sub-regiões. Pesquisa Agropecuária Brasileira, Brasília, v. 33, n. especial, p. 1703-11, out. 1998.

SOUZA, L. R.; HANUS, J. S.; LIBERA, L. B. D.; SILVA, V. M.; MANGILLI, E. M.; SIMÕES, P. W.; CERETTA, L. B.; TUON, L. Sobrecarga no cuidado, estresse e impacto na qualidade de vida de cuidadores domiciliares assistidos na atenção básica. Cadernos Saúde Coletiva, Rio de Janeiro, v. 23, n. 2, p. 140-49, abr./jun. 2015.

STACKFLETH, R.; DINIZ, M. A.; FHON, J. R. S.; VENDRUSCULO, T. R. P.; FABRÍCIO-WHEBE, S. C. C.; MARQUES, S.; RODRIGUES, R. A. P. Sobrecarga de trabalho em cuidadores de idosos fragilizados que vivem no domicílio. Acta Paulista de Enfermagem, São Paulo, v. 25, n. 5, p. 768-74, 2012.

VIANA, R. A. S.; ZUFFI, F. B.; OHL, R. I. B.; CHAVAGLIA, S. R. R. Perfil socioepidemiológico de clientes com limitação de mobilidade e seus cuidadores. Revista Enfermagem, Rio de Janeiro, v. 21, n. 4, p. 439-45, out./dez. 2013.

WORLD HEALTH ORGANIZATION (WHO). World report on disability. Geneva: WHO, 2011.

\section{Sobre os autores:}

Arthur de Almeida Medeiros: Doutor e mestre em Saúde e Desenvolvimento na região Centro-Oeste pela Universidade Federal de Mato Grosso do Sul (UFMS). Especializado em Ativação de Processos de Mudança na Formação Super pela Fundação Oswaldo Cruz (Fiocruz). Atualmente é professor adjunto na UFMS. E-mail: arthur.medeiros@ufms.br, Orcid: http://orcid.org/0000-0002-2192-8823

Micheli Silva Alves: Graduada em Fisioterapia pela Universidade Federal de Mato Grosso do Sul (UFMS). E-mail: michely-mik@hotmail.com, Orcid: http://orcid.org/0000-0002-7431-4569

Wilson de Souza Franco: Graduado em Fisioterapia pela Universidade Federal de Mato Grosso do Sul (UFMS). E-mail: wilsonsouza99@gmail.com, Orcid: http://orcid.org/0000-0001-9045-3465 
